Argonne

ANL-10/26

\title{
DDES and IDDES of Tandem Cylinders
}

Leadership Computing Facility Division 


\begin{abstract}
About Argonne National Laboratory
Argonne is a U.S. Department of Energy laboratory managed by UChicago Argonne, LLC under contract DE-AC02-06CH11357. The Laboratory's main facility is outside Chicago, at 9700 South Cass Avenue, Argonne, Illinois 60439. For information about Argonne and its pioneering science and technology programs, see www.anl.gov.
\end{abstract}

\title{
Availability of This Report
}

This report is available, at no cost, at http://www.osti.gov/bridge. It is also available on paper to the U.S. Department of Energy and its contractors, for a processing fee, from:

U.S. Department of Energy

Office of Scientific and Technical Information

P.O. Box 62

Oak Ridge, TN 37831-0062

phone (865) 576-8401

fax (865) 576-5728

reports@adonis.osti.gov

\begin{abstract}
Disclaimer
This report was prepared as an account of work sponsored by an agency of the United States Government. Neither the United States Government nor any agency thereof, nor UChicago Argonne, LLC, nor any of their employees or officers, makes any warranty, express or implied, or assumes any legal liability or responsibility for the accuracy, completeness, or usefulness of any information, apparatus, product, or process disclosed, or represents that its use would not infringe privately owned rights. Reference herein to any specific commercial product, process, or service by trade name, trademark, manufacturer, or otherwise, does not necessarily constitute or imply its endorsement, recommendation, or favoring by the United States Government or any agency thereof. The views and opinions of document authors expressed herein do not necessarily state or reflect those of the United States Government or any agency thereof, Argonne National Laboratory, or UChicago Argonne, LLC.
\end{abstract}




\section{DDES and IDDES of Tandem Cylinders}

prepared by

A. Garbaruk ${ }^{1}$, M. Shur ${ }^{1}$, M. Strelets ${ }^{1}$, P.R. Spalart ${ }^{2}$, and R. Balakrishnan ${ }^{3}$

'St.-Petersburg State Polytechnic University, St.-Petersburg, Russia

${ }^{2}$ Boeing Commercial Airplanes, Seattle, Washington

${ }^{3}$ Leadership Computing Facility Division, Argonne National Laboratory, Argonne, Illinois

September 2010 


\title{
DDES and IDDES of Tandem Cylinders
}

\author{
A. $\operatorname{Garbaruk}^{1}$, M. Shur ${ }^{2}$, M. Strelets ${ }^{3}$ \\ "New Technologies and Services" (NTS), St.-Petersburg, Russia, 197198; \\ St.-Petersburg State Polytechnic University, St.-Petersburg, Russia, 195251 \\ P. R. Spalart ${ }^{4}$ \\ Boeing Commercial Airplanes, Seattle, Washington, 98124, USA \\ and \\ R. Balakrishnan ${ }^{5}$ \\ Argonne National Laboratory, Argonne, Illinois, 60439
}

\begin{abstract}
The paper presents an overview of the authors' contribution to the BANC-I Workshop on the flow past tandem cylinders (Category 2). It includes an outline of the simulation approaches, numerics, and grid used, the major results of the simulations, their comparison with available experimental data, and some preliminary conclusions. The effect of varying the spanwise period in the simulations is strong for some quantities, and not others.
\end{abstract}

\section{Introduction}

$\mathrm{T}$ he simulations have been carried out in the framework of two recent versions of Detached-Eddy Simulation $(\mathrm{DES})^{1}$, namely, Delayed DES or DDES ${ }^{2}$ and DDES with improved wall-modeling capability or IDDES ${ }^{3}$. In both cases the S-A RANS turbulence model is used as the background model. DDES presents a replacement of the original DES essentially eliminating the possibility of Modeled Stress Depletion or Grid-Induced Separation (Refs. $1,2)$ which DES may suffer from on ambiguous grids in natural DES applications, whereas IDDES is an extension of DDES which combines it with a new RANS-LES hybrid model aimed at Wall Modeling in LES (WMLES). IDDES ensures a different response of the model depending on whether the simulation does or does not have inlet turbulent content. In the first case, it reduces to WMLES (that is, most of the turbulence is resolved, except near the wall) and in the second case (without inlet turbulent content) IDDES performs as DDES. More detailed description of the approaches is contained in the original publications (Refs. 2,3) and in a recent review paper of P. Spalart ${ }^{4}$.

In all the simulations the flow is considered as incompressible.

\section{Numerical Method}

Computations were performed with the use of the in-house NTS $\operatorname{code}^{5,6}$. This is a structured finite-volume CFD code accepting multi-block overset grids of Chimera type. Several implicit flux-difference splitting numerical methods (based on the MUSCL approach) are implemented in the code, and for the incompressible flow considered in the present work we used the method of Rogers and Kwak ${ }^{7}$. The spatial approximation of the inviscid fluxes within this method was performed differently in different grid blocks. In particular, in the outer, Euler, block (see Fig.2 below) the $3^{\text {rd }}$-order upwind-biased scheme was used, whereas in the other blocks, a weighted $5^{\text {th }}$ order upwind-biased $/ 4^{\text {th }}$ order centered, scheme with automatic (solution-dependent) blending function ${ }^{8}$ was employed. The viscous terms of the governing equations were approximated with the $2^{\text {nd }}$ order centered scheme.

\footnotetext{
Paper presented at the AIAA Workshop on Benchmark Problems for Aircraft Noise Computations -I (BANC -I) held June 10-11, 2010, immediately after the 16th Aeroacoustics Conference in Stockholm, Sweden.

${ }^{1}$ Associate Professor

${ }^{2}$ Leading Research Scientist, Associate Professor, AIAA Senior Member

${ }^{3}$ Principal Scientist, Professor

${ }^{4}$ Boeing Senior Technical Fellow, AIAA Member

${ }^{5}$ Computational Scientist at the Argonne Leadership Computing Facility (ALCF)
} 
For the unsteady flows considered in the present work, time-derivatives are approximated with the $2^{\text {nd }}$-order implicit backward differences (three-layer scheme) with dual time-stepping (infinite default pseudo-time step) and sub-iterations.

In the Euler block, at every iteration the discretized equations are solved with the use of the diagonally dominant approximate factorization (DDADI) and in the other blocks with the use of the Gauss-Seidel relaxation by planes.

The parallelization of the code is based on a "hybrid" conception which combines MPI and Open MP technologies. This approach permits adjusting the code to the specific hardware being used (shared, distributed or mixed memory structure).

\section{Problem Description}

\section{A. Geometry and Flow Conditions}

The experimental set-up in the BART experiment ${ }^{9}$ and a schematic of the flow in an XY-plane are shown in Fig. 1.
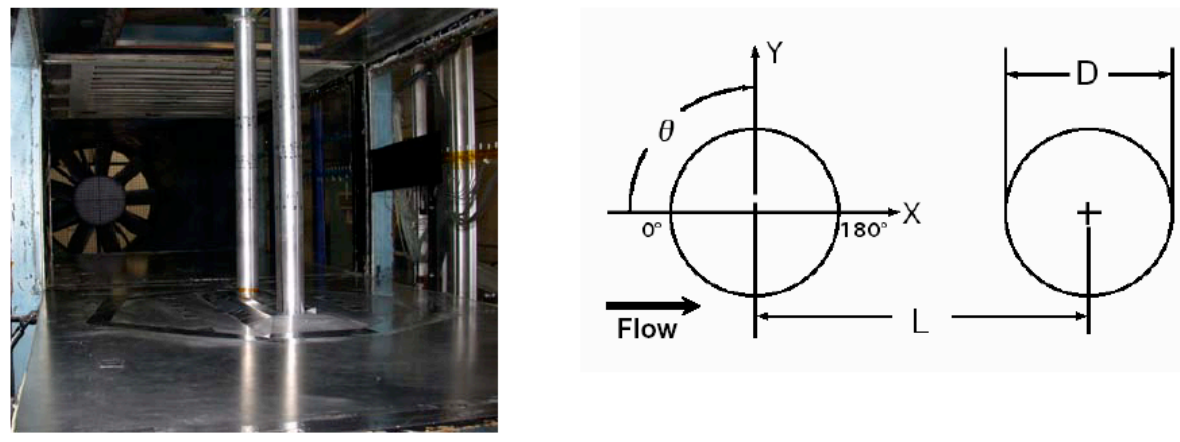

Fig. 1. Photograph of experimental set-up and schematic of the flow in the $X Y$ plane

Specific simulations were performed at the experimental value of the Reynolds number based on the free stream velocity, $V_{0}$, and cylinder diameter, $D$, equal to $\operatorname{Re}_{\mathrm{D}}=1.66 \cdot 10^{5}$, the distance between the cylinder centers $L=3.7 D$, and the distance between the side walls of the experimental section (in the $y$-direction in Fig.1), $L_{\mathrm{y}}$, equal to $22.86 D$. For the spanwise, $z$, direction, the flow was assumed to be homogeneous which motivates imposing periodic conditions in this direction. Two simulations (DDES and IDDES) were performed in a domain with the span size $L_{\mathrm{z}}=3 D$ and one DDES simulation was carried out also with $L_{\mathrm{z}}=16 D$ (the latter was run on the Intrepid supercomputer of Argonne National Laboratory). The remaining boundary conditions were as follows.

At the inflow, uniform streamwise and zero lateral velocity components were specified and the eddy viscosity was set equal to the molecular one $\left(v_{t} / v=1.0\right)$. The latter condition implies the fully turbulent approach to transition control (immediate transition in all boundary layers) and is justified at least for the upstream cylinder since its boundary layer has been tripped in the BART experiment ${ }^{1}$.

On the surface of the cylinders the no-slip and non-permeability conditions were imposed for velocity, and the eddy viscosity was set equal to zero. The side-walls of the wind tunnel section were assumed to be free-slip boundaries.

Finally, at the outflow a constant pressure was specified and velocity was linearly extrapolated from the interior of the domain.

The time step in DDES and IDDES at $L_{\mathrm{z}}=3 D$ was $0.02 D / V_{0}\left(2.6 \cdot 10^{-5} \mathrm{~s}\right)$, and in DDES at $L_{\mathrm{z}}=16 D$ it was $0.005 \mathrm{D} / V_{0}\left(6.5 \cdot 10^{-6} \mathrm{~s}\right)$. These values ensure a CFL number of less than one in the most sensitive areas of the flow (the gap between the cylinders and the near wake of the back cylinder).

The simulations were initialized from a uniform flow or restarted from available fields from another simulation and ran for about 350 convective time units, $D / \mathrm{V}_{0}$, with 300 units only used for computing the mean flow and turbulence statistics. An illustration of a typical convergence accuracy of the turbulent statistics is given in Fig. 2, which shows the rms of the pressure coefficient fluctuations on the upstream and downstream cylinders computed based on times samples of 100, 200, and 300 convective time units, respectively, and a running time-average of the

\footnotetext{
${ }^{1)}$ For the downstream cylinder, only limited experimental data with its tripping are available, and this should be kept in mind
} when comparing results of the simulations with the data (see discussion in the next section). 
integral drag coefficients of the upstream and downstream cylinders (the "final" values, after averaging over 300 convective time units, of the drag are presented in Table 1).
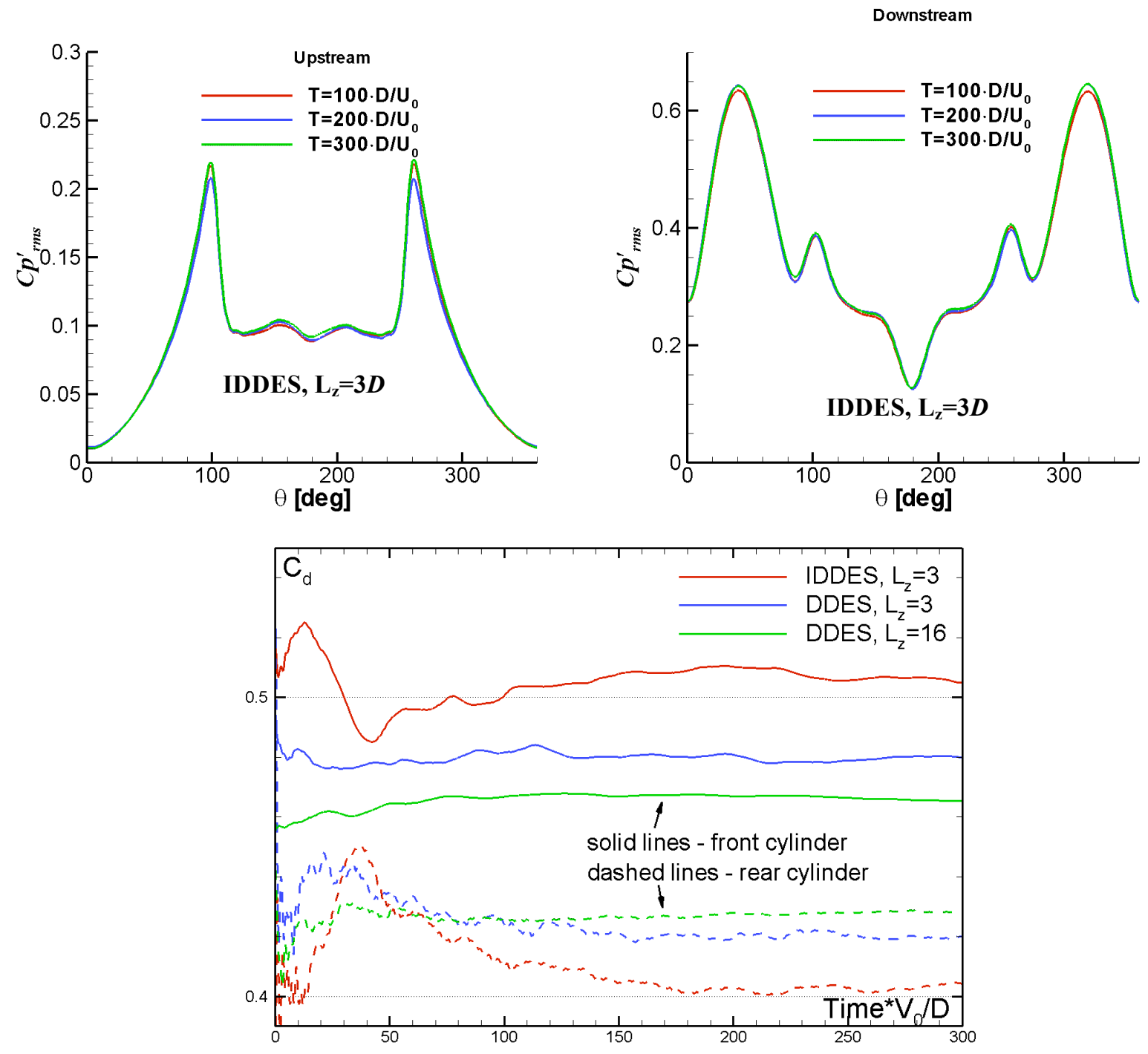

Fig.2. RMS of pressure coefficient fluctuations on the cylinders (upper frames) and running average of the integral drag coefficient (lower frame)

Table 1

\begin{tabular}{|c|c|c|c|}
\hline Approach & $\boldsymbol{L}_{\mathbf{z}} / \mathbf{D}$ & Upstream cylinder & Downstream cylinder \\
\hline IDDES & 3 & 0.51 & 0.40 \\
\hline DDES & 3 & 0.48 & 0.42 \\
\hline DDES & 16 & 0.47 & 0.43 \\
\hline
\end{tabular}

\section{B. Grids}

The $X Y$ grid used in all the simulation is shown in Fig.3. It is designed in accordance with the guidelines for DESlike simulations ${ }^{10,11}$ and has 5 blocks: 1 block in the outer or Euler Region (ER), 3 blocks in the Focus Region (FR), which includes the gap between the cylinders and the near wake of the downstream cylinder, and 1 block in the Departure Region (DR). The total number of nodes on the surface of the upstream cylinder is 245 (the distance between the nodes on the upstream half of this cylinder is close to $0.02 D$ and on its downstream part it is $0.01 D$ with smooth transition between the two). On the downstream cylinder there are 380 uniformly distributed nodes (the 
distance between the nodes is $0.008 D$ ). The $r$-step closest to cylinders' walls, in the wall units, is less than 1.0. In the major part of the FR the cells are nearly isotropic with a size of about $0.02 D$. In the ER and DR the grid steps increase gradually (linearly with $r$ ) . The total size of the grid in the $X Y$ plane is 82,000 cells.

The spanwise grid is uniform with a step $\Delta z=0.02 D$. Thus, the total number of cells is 11 million for the simulations with $L_{\mathrm{z}}=3 D$, and 60 million with $L_{\mathrm{z}}=16 D$.
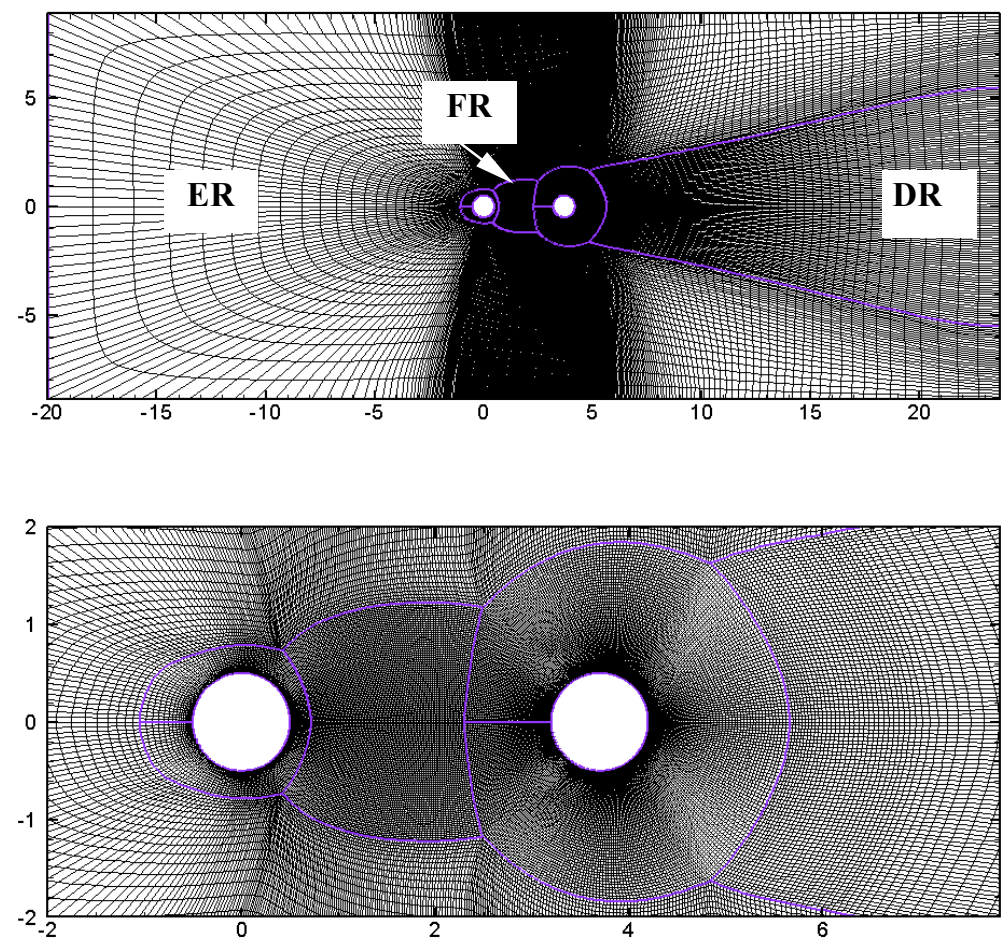

Fig. 3. Grid in the XY plane used in the simulations, and zoomed fragment

\section{Results}

In this section we briefly present results of the simulations described above.

The sufficiently high quality in the LES region provided by the numerics and grid used in the present simulations, even somewhat beyond the FR is supported by Fig.4, which shows the PSD spectra of the streamwise velocity at the sample point indicated in the left frame of the figure by a yellow circle: the extent of the inertial range of frequencies in the spectra is about a whole decade in all the three simulations. This strongly suggests the existence of a proper three-dimensional energy cascade. The Karman-street shedding peak near a Strouhal number of 0.25 , as is typical with turbulent separation and observed in the experiment (see Fig.15 below), is also evident.
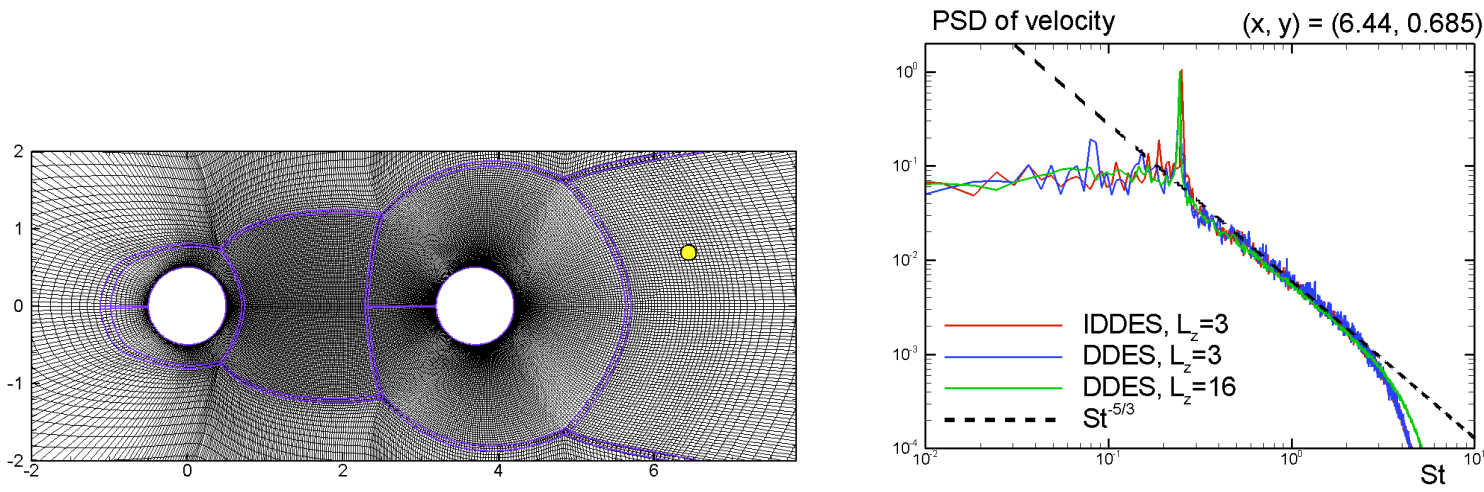
Fig. 4. Power Spectral Density (PSD) spectrum of velocity from simulations (right frame) at the point shown in the left

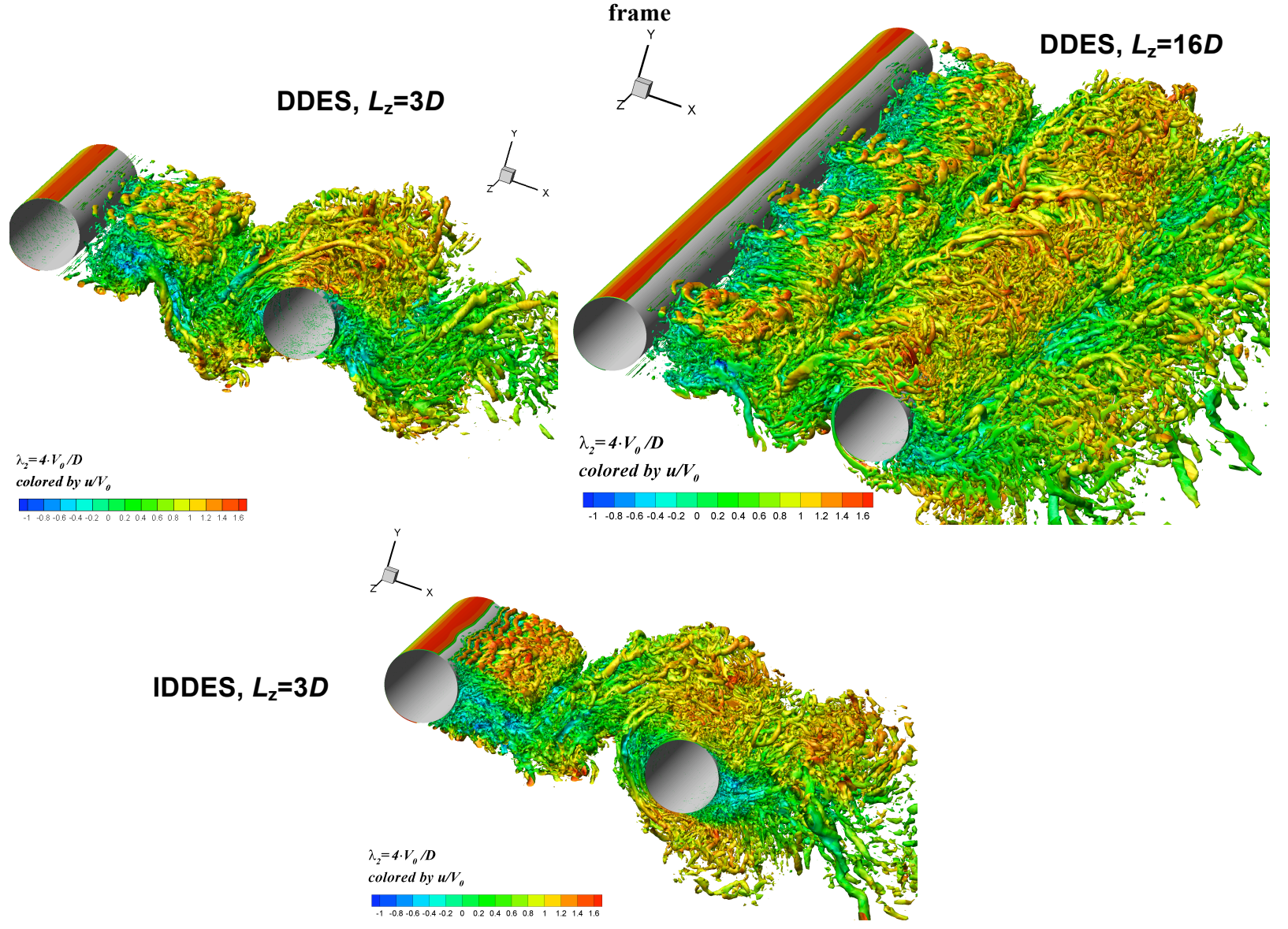

Fig. 5. Isosurface of swirl $\lambda=4.0 V_{0} / D$ from three simulations

Figure 5 presents sample flow visualizations (instantaneous isosurface of the magnitude of the second eigen value of the velocity gradient tensor, or swirl quantity) from the three simulations. It visibly displays the capability of the simulations to resolve fine-grained turbulence (consistent with the grid used), and exhibits the complex although in general, similar vortical structures predicted by the different simulations. At the same time it reveals some difference between the three simulations, especially in the region of the flow separation from the upstream cylinder. One can see, in particular, that the elongation of the span size of the computational domain in DDES from $3 D$ up to $16 D$ causes a delay of the onset of the shear layer instability and roll-up, whereas moving from DDES to IDDES at the same span size of the domain $L_{\mathrm{z}}=3 D$ leads to the opposite effect (tangibly earlier onset of the instability). Note that the domain size $3 D$ has been typical of cylinder simulations and sufficient for fair agreement on basic quantities such as the drag coefficient ${ }^{12}$, whereas $16 \mathrm{D}$ represents an intensified effort to duplicate the much longer cylinders used in experiments.

The same trends are seen in Fig. 6 which compares instantaneous contours of the spanwise vorticity in an $X Y$ plane predicted by the three simulations with each other and with the PIV measurements ${ }^{9}$. Moreover, the figure displays a qualitatively similar, although less pronounced, difference between the simulations in the shear layers separated from the downstream cylinder. Thus, the wake of the second cylinder appears less challenging. As far as agreement with experiment is concerned, although based on the instantaneous vortical patterns, it is difficult to give a definite preference to any of the simulations, it seems that the DDES prediction at $L_{\mathrm{z}}=3 D$ is somewhat closer to the experiment than those of DDES at $L_{\mathrm{z}}=16 D$ and IDDES at $L_{\mathrm{z}}=3 D$. However, considering that the simulation in a wide domain is more representative "by definition" and that the IDDES approach is generally expected to be more accurate, this brings up the conjecture that the apparently better performance of DDES at $L_{\mathrm{z}}=3 D$ is a result of cancellation between modeling errors and those associated with the insufficient span size of the domain. The difference between fully-turbulent CFD and a tripped experimental flow could also play a role. This comparison may reveal subtleties which have not been covered in the literature, before this workshop. 

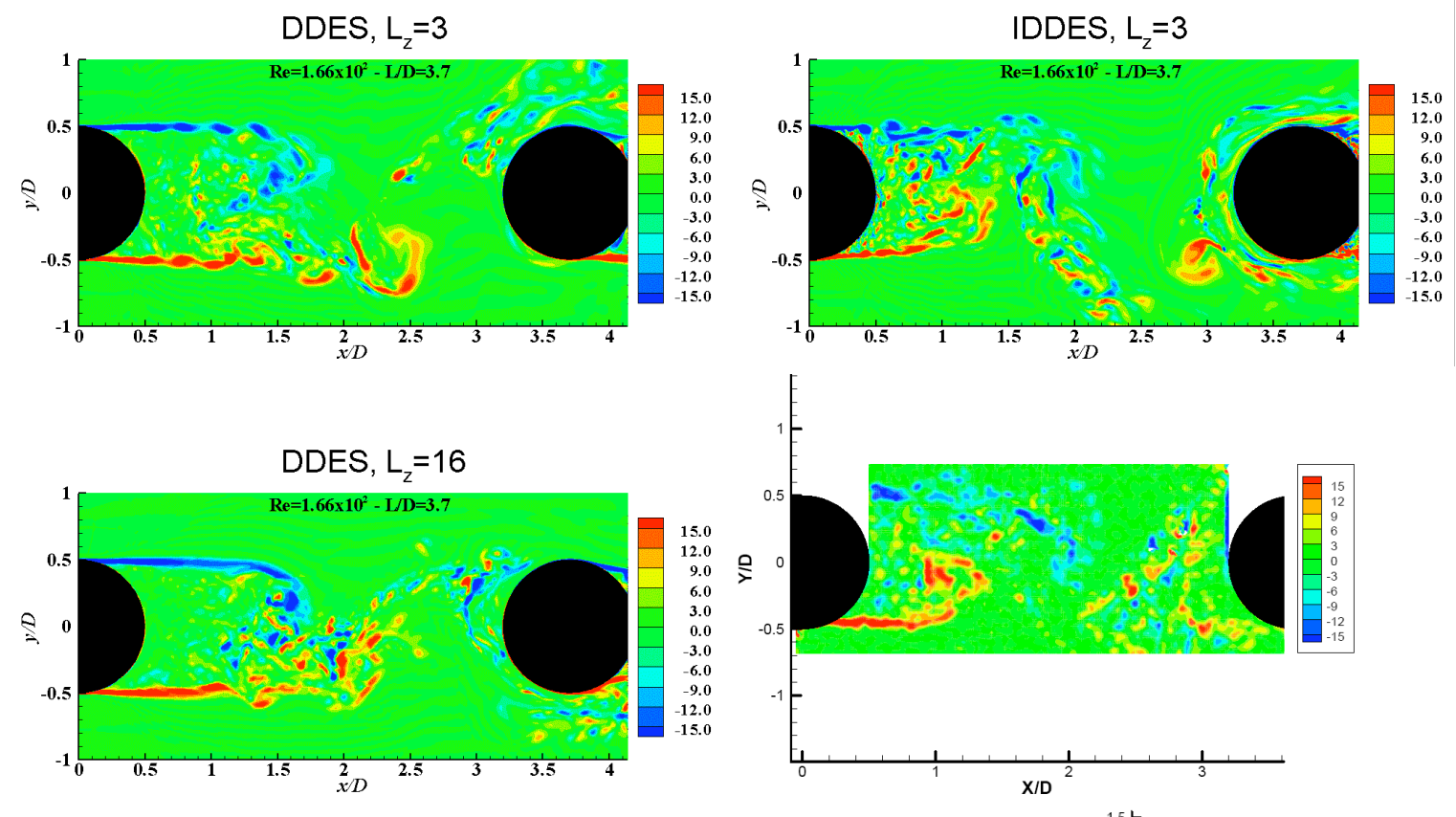

DDES, $L_{2}=3$
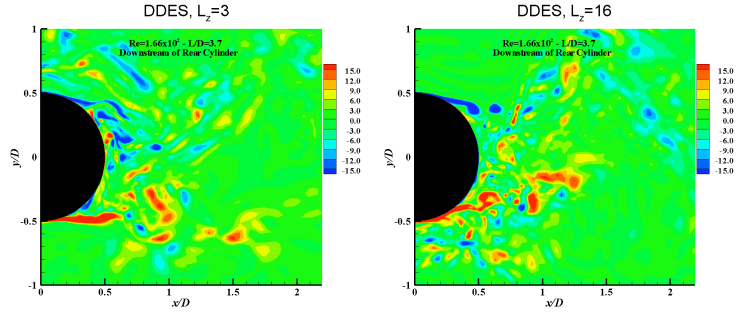
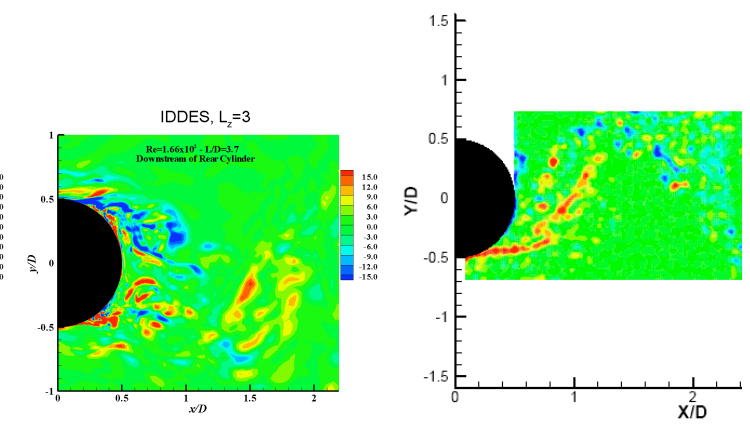

Fig. 6. Comparison of contours of spanwise vorticity component from simulations with PIV measurements

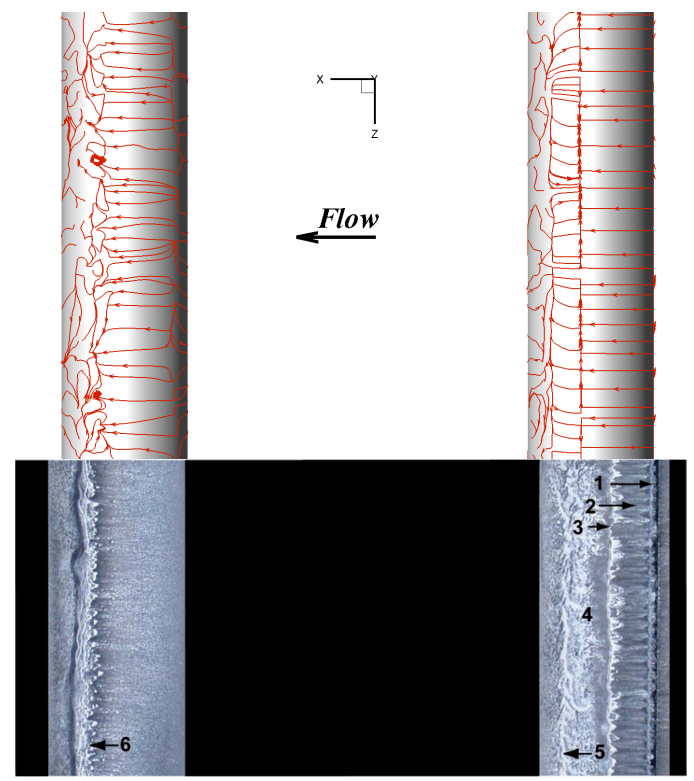

Fig. 7. Comparison of oil-flow patters from DDES at $L z=16 D$ with experiment: 1- transition strip;

2-streaks from streamwise vortices generated by transition strip; 3- primary separation line; 4- spanwise flow between 2 separation lines; 5- secondary separation line; 6- rear cylinder separation. NOTE: Experimental view is a bit distorted due to finite distance to the camera 
Another flow visualization, now in the form of the oil-flow from the experiment and DDES at $L_{z}=16 D$ is shown in Fig.7. Based on this figure, for the upstream cylinder, the simulation predicts a somewhat delayed primary separation (which could be related to tripping and especially over-tripping, or else simply be a failing of the RANS model), and a correct location of the secondary separation (lines 3 and 5 in the figure respectively). For the downstream cylinder, the CFD and experimental oil-flow patters agree very well.

Let us now consider in Figs. 8-15 a more quantitative comparison of the results of the simulations with measurements.
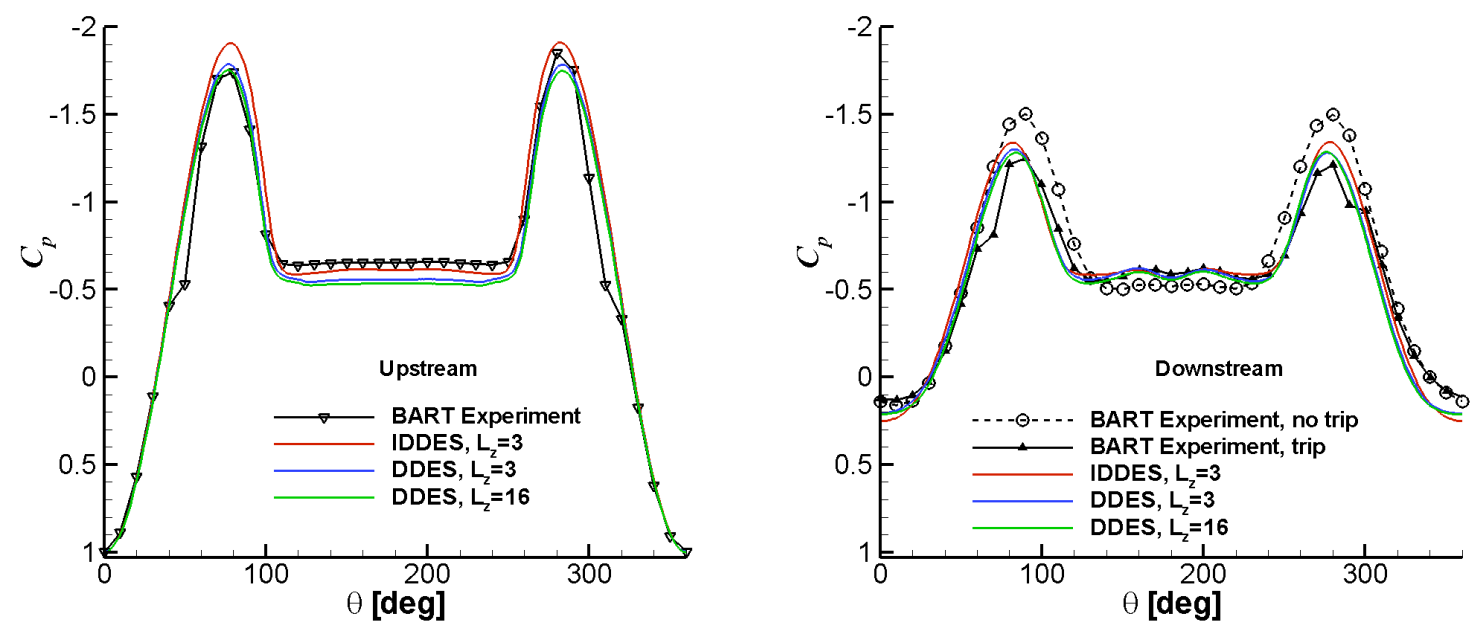

Fig. 8. Surface pressure distributions over the upstream (left) and downstream (right) cylinders

Figure 8 shows such a comparison of distributions of the pressure coefficient $C_{p}$ on both cylinders. Note that for the downstream cylinder two sets of data are available, one from the experiment with tripping of only the upstream cylinder and the other with tripping of both cylinders, the latter being a more appropriate arrangement to compare with the numerical predictions based on the FT approach to transition control. The first conclusion based on this figure is that both DDES computations predict virtually identical $C_{\mathrm{p}}$ distributions and that the difference between DDES and IDDES at $L_{\mathrm{z}}=3 D$ is slight. As for the agreement of the simulations with the measurements, it is fairly good and, as could be expected, for the downstream cylinder it is better in the case with tripping of its boundary layer in the experiment (triangle symbols in the right frame of Fig. 8). Thus, judging by the mean pressure distributions, the results of all the simulations may be considered as quite satisfactory. This confirms earlier findings in Ref. 12.
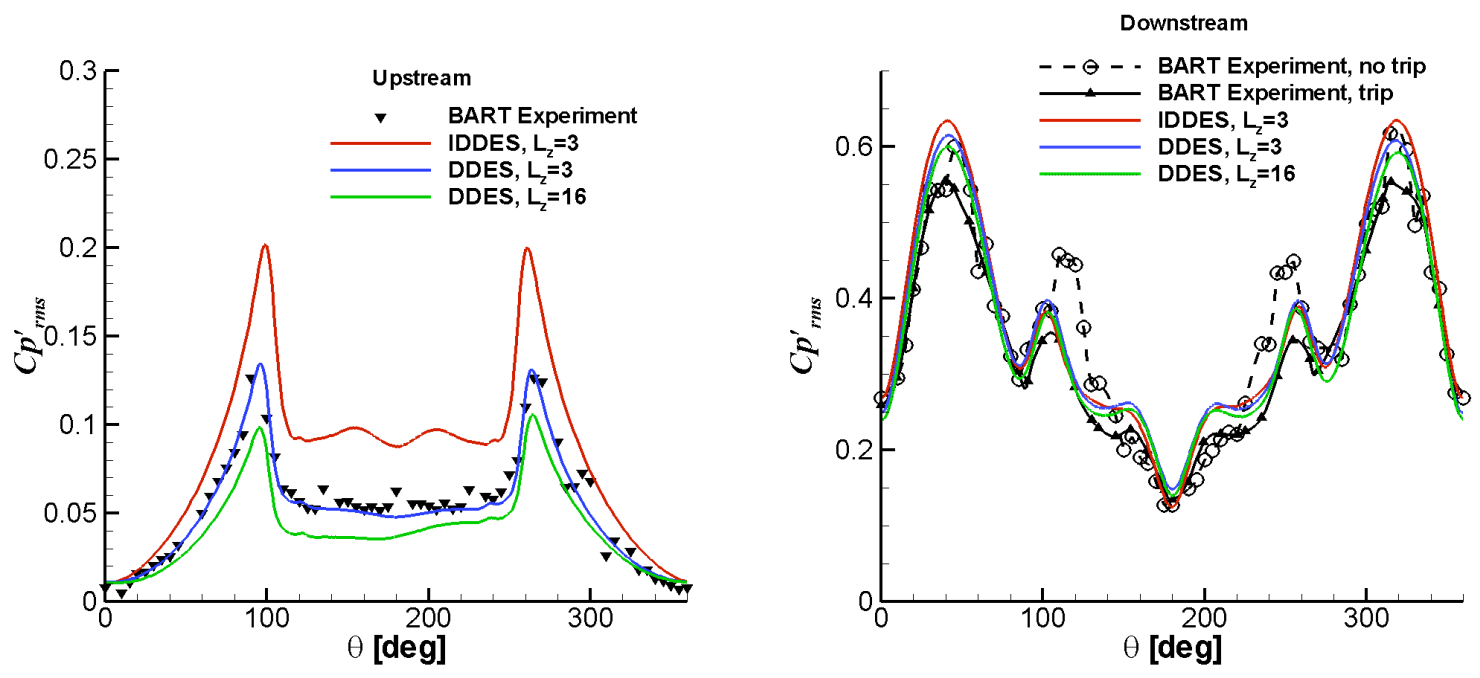
Fig. 9. Surface distributions of rms of pressure coefficient on the upstream (left) and downstream (right) cylinders

With respect to the rms of pressure coefficient, the situation is not so favorable. As seen in Fig. 9, its computational distribution on the upstream cylinder turns out to be highly sensitive to both the span-size of the domain and the simulation approach. In particular, at $L_{\mathrm{z}}=3 D$ DDES predicts tangibly lower pressure fluctuations than IDDES, and agrees with the experiment fairly well. On the other hand, at $L_{\mathrm{z}}=16 D$, which logically is a superior simulation, $C_{P}^{\prime}$ as predicted by DDES becomes quite a bit lower than the data. This behavior seems to be consistent with the different predictions of the onset of instability of the shear layer separated from the upstream cylinder discussed above regarding the flow visualizations in Figs. 5, 6. Thus, if the error cancellation conjecture formulated there is correct, it should be expected that an IDDES carried out in the wide domain (a simulation which is in progress now) has a good chance of providing rather accurate prediction of the $C_{P}^{\prime}$ distribution on the upstream cylinder. For the downstream cylinder, again consistently with the flow visualizations shown in Figs. 5, 6, no significant difference between the three simulations is observed and, just as for the pressure coefficient itself, the agreement with the data obtained with tripping of this cylinder is, in general, somewhat better than with the data without tripping.
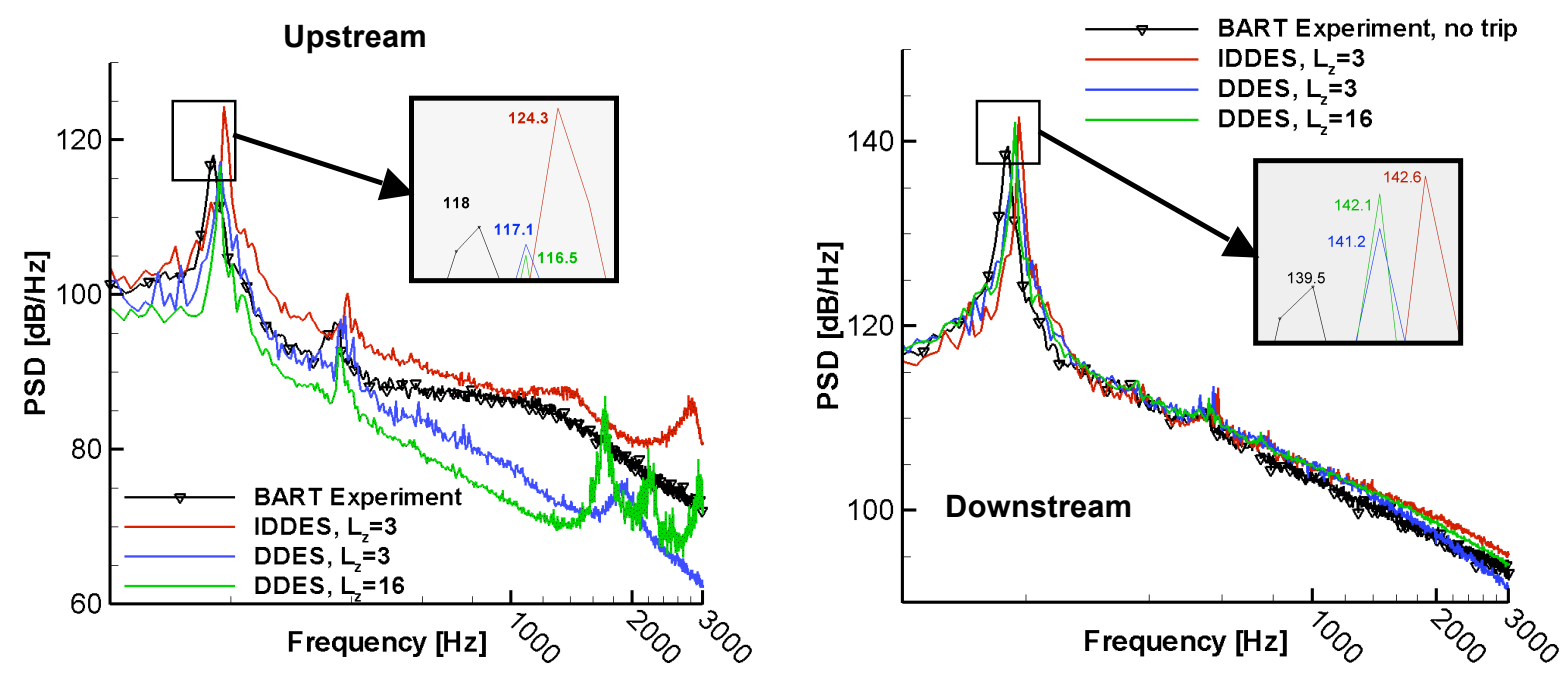

Fig. 10. Power Spectral Density of the surface pressure at 135 degrees for the upstream and at 45 degrees for the downstream cylinders

Surface pressure PSD spectra from the simulations presented in Fig. 10 reveal peaks at the shedding frequency and its harmonics. The frequency is within 5\% of the experimental value (see Table 2). As for the amplitude of the main peak, for the upstream cylinder it is somewhat underestimated by DDES and tangibly overestimated by IDDES. These trends, again, are consistent with the flow visualizations, and this quantity appears to be extremely sensitive. For the downstream cylinder, all the three simulations overestimate the peak amplitude, but when comparing the predicted and experimental spectra for this cylinder one should keep in mind that the latter are available only in the experiments without tripping. Note that for the upstream cylinder all the simulations predict high-frequency peaks which are more pronounced in the DDES carried out in the wide domain. These peaks are not seen in the experimental spectra and are probably associated with the initial shear layer roll-up. In general, the spectra support the conclusions formulated above for the rms of pressure coefficient and, in particular, reveal a relatively strong sensitivity of the pressure fluctuations on the upstream cylinder to both the span-size of the domain and the simulation approach used. In contrast to this, the spectra on the downstream cylinder are much less sensitive to either the span extent or the approach (DDES or IDDES) and agree reasonably well with the data.

Table 2

\begin{tabular}{|c|c|c|}
\hline Simulation approach & Shedding frequency, $\mathbf{H z}$ & Experiment, $\mathbf{H z}$ \\
\hline DDES, $\mathbf{L z}=$ 3D & 188 & \multirow{2}{*}{$\mathbf{1 7 8}$} \\
\hline DDES, $\mathbf{L z}=\mathbf{1 6 D}$ & 188 & \\
\hline IDDES, $\mathbf{L z}=\mathbf{3 D}$ & 192.5 & \\
\hline
\end{tabular}


The last surface turbulence characteristic available in the experimental database is the two-point spanwise pressure correlation, $R_{\mathrm{pp}}$. A comparison of predicted spanwise variation of $R_{\mathrm{pp}}$ with the data is presented in Fig. 11 . For the upstream cylinder the computed correlation first follows the experimental curve and then starts deviating from it, at $\Delta_{\mathrm{z}} / D \approx 1$ for the both simulations carried out at $L_{\mathrm{z}}=3 D$ and at $\Delta_{\mathrm{z}} / D \approx 3$ for DDES performed at $L_{\mathrm{z}}=16 D$. In the latter case it remains as high as about 0.25 (whereas, of course, the ideal behavior with a long-enough period is to drop to 0 ), and in the experiment is drops to zero for $\Delta_{Z} / D \approx 6$. For the downstream cylinder, the behavior of the computed correlation coefficient in the wide domain is quite different: it virtually coincides with that in the narrow domain at small $\Delta_{\mathrm{Z}} / D$, and then continues to decrease much more slowly than in the experiment so that at $\Delta_{\mathrm{Z}} / D \approx 6$, where the experimental values are close to zero, it remains as high as $\sim 0.55$. Although concrete reasons of these discrepancies are not clear, the behavior of the computed correlation coefficients is consistent with the flow visualizations in Fig. 5, which reveal the presence of a global quasi-2D shedding in the simulations. The drift of the shedding phase versus $z$ is small. Thus, even at $L_{\mathrm{z}}=16 D$, imposing periodic boundary conditions does not seem to be fully justified. Naturally, imposing lateral walls at $16 \mathrm{D}$ does not either represent the ideal situation, in which the length of the model is so large that it has no influence at all any more. The complete situation would become even more complex if we envisioned using initial conditions with oblique vortex shedding, a phenomenon which is wellknown at lower Reynolds numbers. After seeing Fig. 5, it is easy to imagine that oblique shedding with a period of $16 D$ would be very viable.
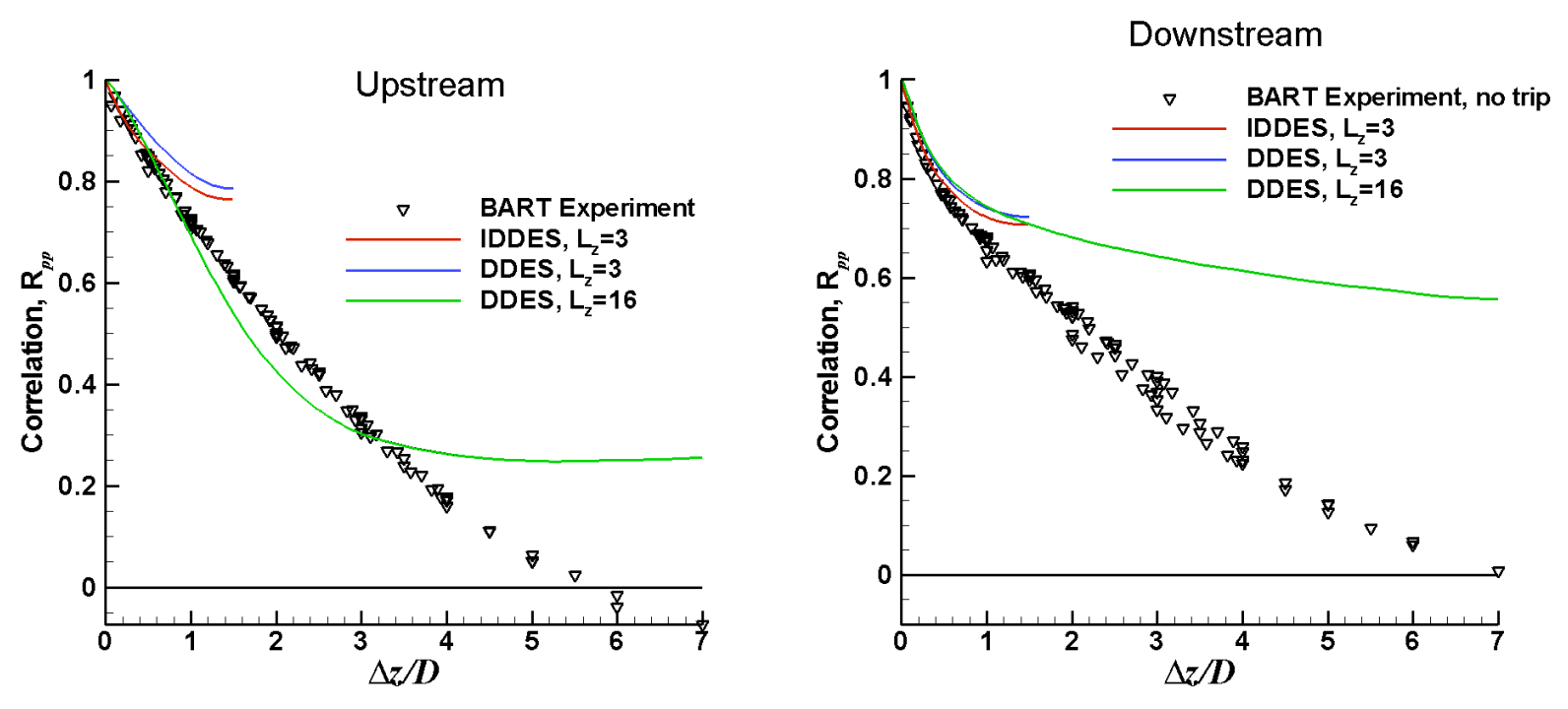

Fig. 11. Surface pressure correlation at azimuthal angle $\theta=135^{\circ}$ for the upstream cylinder (left) and $\theta=45^{\circ}$ for the downstream cylinder (right)

Let us now dwell upon the comparison of predicted and available measured characteristics of the flow (centerline distributions of the mean velocity and 2D turbulent kinetic energy and profiles of kinetic energy) in the gap between cylinders and in the wake of the downstream cylinder, as presented in Figs.12-14. As seen in the figures, none of the simulations predicts all these characteristics accurately enough by modern standards, but still DDES results agree with the data tangibly better than those of IDDES. Other than that, the sensitivity of the DDES predictions to the span size of the domain $(3 D$ or $16 D)$ is observed only in the gap between the cylinders (in the wake of the downstream cylinder, they are virtually identical with the two values of $L_{\mathrm{z}}$ ). Finally, when comparing the simulations with the data in the downstream cylinder wake, one should keep in mind that at least partially the disagreement can be caused by the absence of tripping of this cylinder's boundary layer in the experiment. 

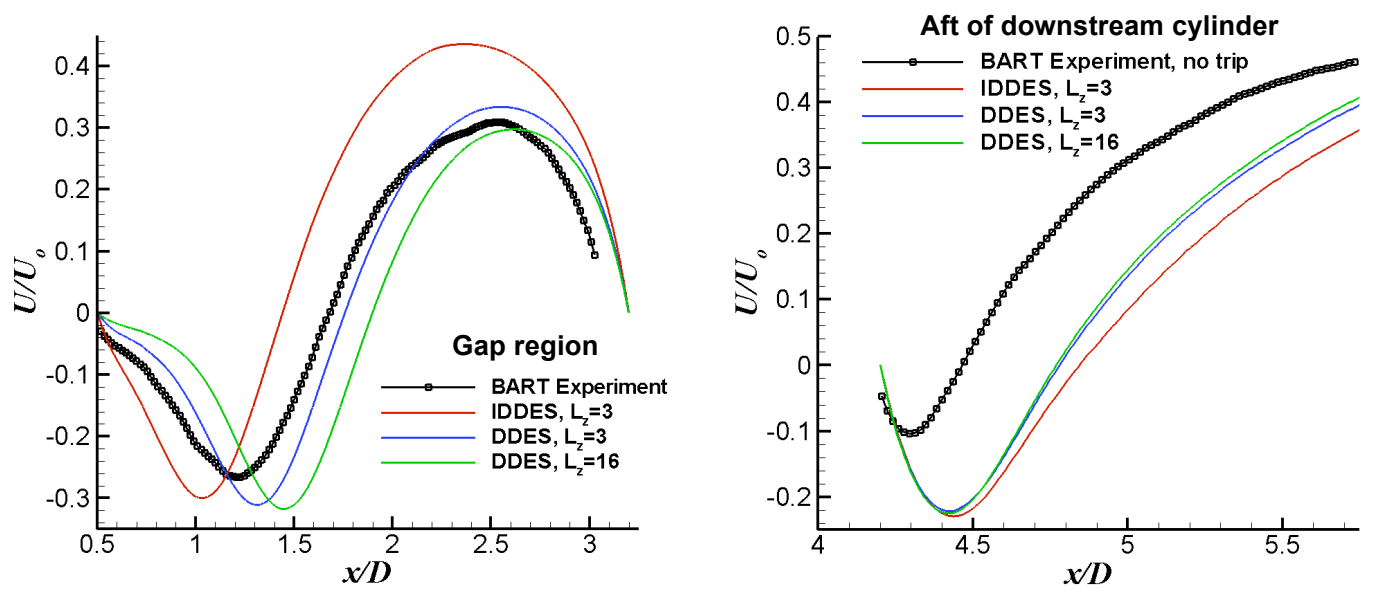

Fig. 12. Distribution of time-averaged streamwise velocity along the symmetry plane $y=0$
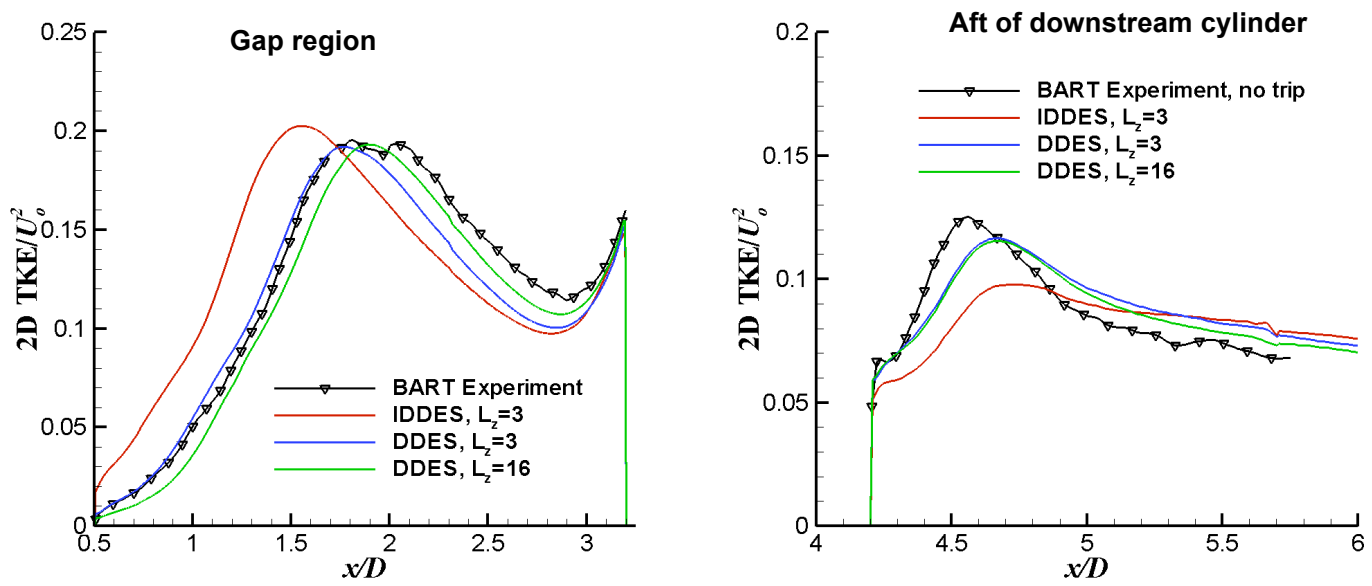

Fig. 13. Distribution of 2D TKE along the symmetry plane $y=0$
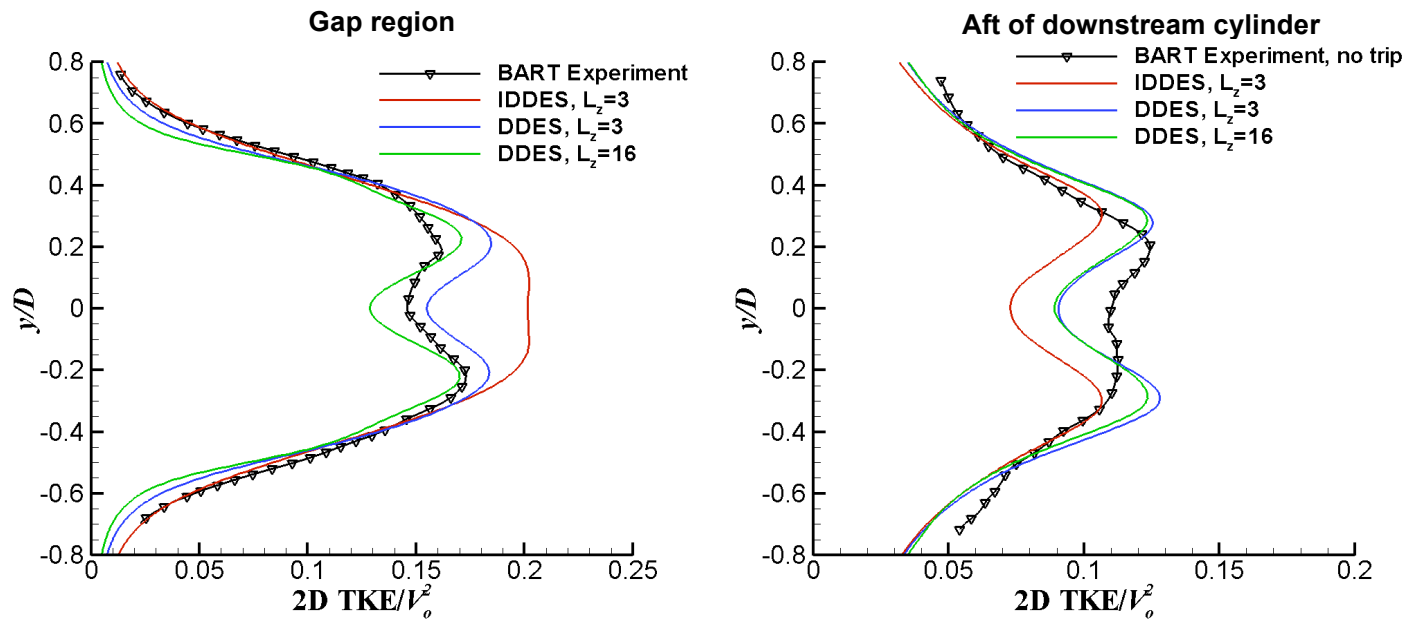

Fig.14. Profiles of 2D TKE in the gap region $(x / D=1.5)$ and in the downstream cylinder's wake $(x / D=4.45)$

Finally, Fig. 15 (its left frame is a zoomed fragment of the spectrum in Fig. 4) compares PSD velocity spectra at the field point $(x, y)=(6.44 .0 .684)$, also shown in Fig. 4 with the experimental spectrum at the same point. It 
suggests that in the mid-wake area of the downstream cylinder all the three simulations predict virtually identical velocity fluctuations which are furthermore close to those observed in the experiment.
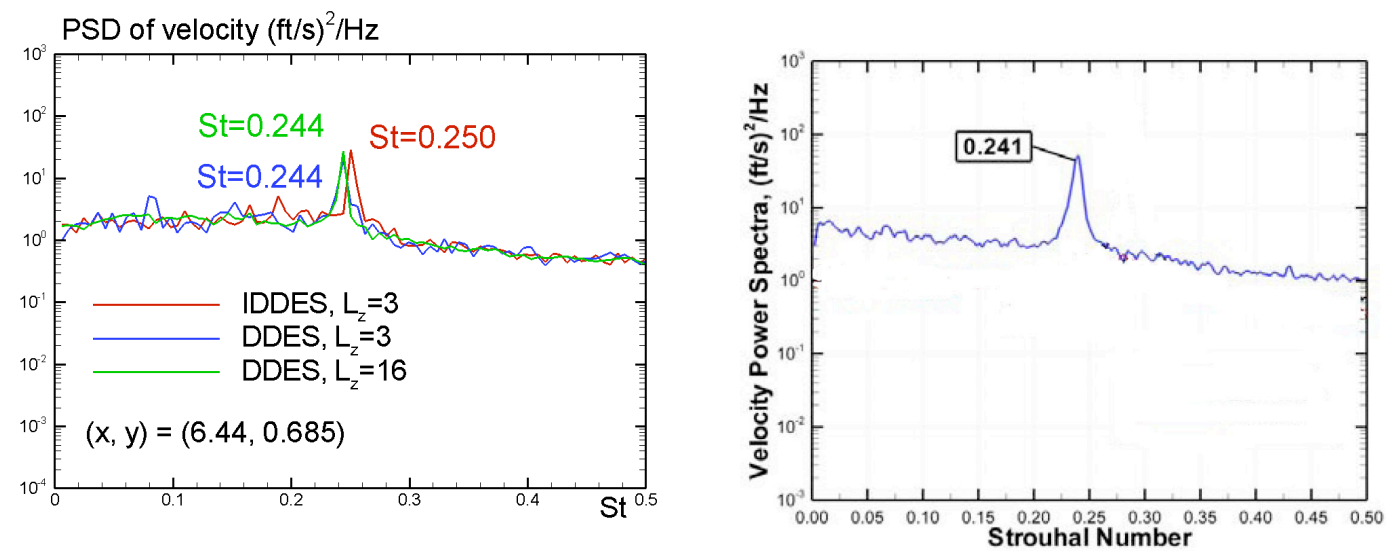

Fig. 15. Comparison of zoomed fragments of PSD spectra of velocity from simulations (left frame) and experiment (right frame) in the mid-wake of the downstream cylinder

\section{Resources}

Both DDES and IDDES simulations carried out with $L_{\mathrm{z}}=3 D(11 \mathrm{M}$ cells $)$ ran on 4 nodes of the NTS PC cluster. One node of this cluster has two 4-core processors Intel Xeon E5345 (2.33GHz) and 8Gb memory. The internodes communication is supported by a gigabit local network.

The DDES $L_{\mathrm{z}}=16 D(60 \mathrm{M}$ cells) was conducted on 8,160 nodes of the Intrepid computer (Argonne National Laboratory, Blue Gene/P architecture), in which each node has as its processor an IBM PowerPC $450(850 \mathrm{MHz})$ and $2 \mathrm{~Gb}$ memory; the internodes communication is supported by a Blue Gene Network.

The CPU and memory resources for both types of simulations are summarized in Table 3.

Table 3

\begin{tabular}{|l|l|l|l|l|l|l|}
\hline & $\begin{array}{l}\text { Wall Clock } \\
\text { Time/time- } \\
\text { step }\end{array}$ & $\begin{array}{l}\text { Number of } \\
\text { time-steps in } \\
\text { simulation }\end{array}$ & $\begin{array}{l}\text { Wall clock } \\
\text { Time/1 sec of } \\
\text { simulation time }\end{array}$ & $\begin{array}{l}\text { Number of time steps } \\
\text { needed for 1 sec of } \\
\text { simulation time }\end{array}$ & $\begin{array}{l}\text { Memory } \\
\text { per cpu }\end{array}$ & $\begin{array}{l}\text { Total } \\
\text { memory }\end{array}$ \\
\hline $\begin{array}{l}\text { NTS Cluster (DDES } \\
\text { and IDDES at } \boldsymbol{L}_{\mathbf{z}}=\mathbf{3 D} \text { ) }\end{array}$ & $2.5 \mathrm{~min}$ & 17,500 & 66 days & 38,500 & $2 \mathrm{~Gb}$ & $8 \mathrm{~Gb}$ \\
\hline $\begin{array}{l}\text { Intrepid (DDES at } \\
\boldsymbol{L}_{\mathbf{z}}=\mathbf{1 6} \boldsymbol{D} \text { ) }\end{array}$ & $12 \mathrm{~s}$ & 78,000 & 22 days & 154,000 & $2 \mathrm{~Gb}$ & $16 \mathrm{~Tb}$ \\
\hline
\end{tabular}

\section{Conclusion}

The work briefly presents results of the incompressible simulations of the tandem cylinder flow carried out in the framework of two relatively recent versions of Detached-Eddy simulation (DES), namely, Delayed DES (DDES) and DDES with Improved wall modeling capability (IDDES). In both cases, the fully turbulent approach is applied for transition control. The DDES runs are performed in two (narrow and wide) computational domains $\left(L_{\mathrm{z}}=3 D\right.$ and $16 D$ respectively), whereas IDDES has so far been accomplished only in the narrow domain. The accumulated experience allows us to describe the flow as a serious challenge for CFD and turbulence modeling. The simulations suggest that a major physical and computational problem is associated with a correct representation of the onset of instability in the turbulent shear layer forming after separation of the turbulent boundary layer from the upstream cylinder. It could be argued that the mission of a RANS turbulence model in such a layer is debatable, in the sense that Kelvin-Helmholtz roll-up may or may not be part of the desired behavior. Exactly in this region, the unsteady flow characteristics are most sensitive to the simulation approach and span size of the computational domain, and it may well be that this issue cannot be resolved other than by imposing a realistic turbulent content in the boundary layer upstream of the separation, in other words, at a far higher level of computing cost than even the higher one which was accepted here, on an especially powerful machine by 2010 standards.

In terms of agreement with experiment, simulations performed do not permit to give a definite preference to one of the two approaches used here to turbulence representation. Although the results of DDES carried out with $L_{\mathrm{z}}=3 D$ 
agree with the data reasonably well and, in general, tangibly better than those of the two other simulations, an appropriately skeptical analysis of the results justifies the conjecture that this advantage may be actually caused by a cancellation between modeling errors and those associated with the insufficient spanwise size of the computational domain. If this conjecture is correct, the IDDES in a sufficiently wide domain has much potential. It should be mentioned also that, although the grid and numerics used in the simulations seem to be "good enough", a substantial grid-refinement may well also change the current assessments.

No attempt to predict the noise generated by the tandem has been undertaken so far, even as the ultimate goal of this work is noise prediction. However considering that much noise is generated by the downstream cylinder and that predicted unsteady pressures on this cylinder are not very sensitive to simulation approach and span-size of the computational domain, it seems that prediction of the noise with the use of reliable CAA tools (e.g., FWH based ones) has a good chance to be successful (not ignoring the usual problem of scaling the noise to different lengths of cylinder).

Finally, in terms of the suitability of the experimental set-up to the validation of the CFD / CAA approaches, the lack of tripping of the downstream cylinder in most of the experiments is somewhat unfavorable, as the potential of convected turbulence, which does not contain very small scales, to trigger boundary-layer transition is unknown. Other than that, measurements of the skin friction distribution over both cylinders would be very desirable in order to evaluate the accuracy of the predicted separation points.

\section{Acknowledgments}

This research used resources of the Argonne Leadership Computing Facility at Argonne National Laboratory, which is supported by the Office of Science of the U.S. Department of Energy under contract DE-AC0206CH11357. The authors of this paper gratefully acknowledge the computing time on the Blue Gene/P at Argonne National Laboratory (Intrepid), which was made available via the Director's Discretionary Allocation. This work was funded by The Boeing Company and, partially, by the EU project ATAAC, Contract No. ACP8-GA-2009233710-ATAAC, and by the Russian Basic Research Foundation (grant No. 09-08-00126).

\section{References}

${ }^{1}$ Spalart, P.R., Jou, W.-H., Strelets, M., and Allmaras, S. R., "Comments on the feasibility of LES for wings, and on a hybrid RANS/LES approach," 1st AFOSR Int. Conf. on DNS/LES, Aug. 4-8, 1997, Ruston, LA. In: “Advances in DNS/LES,” C. Liu and Z. Liu (Eds.), Greyden Press, Columbus, OH.

${ }^{2}$ Spalart, P. R., Deck, S., Shur, M. L., Squires, K. D., Strelets, M. Kh., Travin, A., K., “A new version of Detached-Eddy Simulation, resistant to ambiguous grid densities," Theor. Comp. Fluid Dyn. Vol. 20, No. 3, 2006, pp. 181-195.

${ }^{3}$ Shur, M. L., Spalart, P. R., Strelets, M. Kh., Travin, A., "A hybrid RANS-LES model with delayed DES and wall-modeled LES capabilities," Int. J. Heat Fluid Flow, Vol. 29, No. 6, 2008, pp. 1638-1649.

${ }^{4}$ Spalart P.R., "Detached Eddy Simulation," Annual Review Fluid Mechanics, Vol. 41, pp. 181-202.

${ }^{5}$ Strelets, M. Kh., "Detached Eddy Simulation of Massively Separated Flows," AIAA Paper, AIAA-2001-0879, 2001.

${ }^{6}$ Shur, M., Strelets, M., Travin, A., "High-Order Implicit Multi-Block Navier-Stokes Code: Ten-Years Experience of Application to RANS/DES/LES/DNS of Turbulent Flows," Invited lecture. 7th Symposium on Overset Composite Grids and Solution Technology, October 5-7, 2004, Huntington Beach, USA

${ }^{7}$ Rogers, S. E., and Kwak, D., "An Upwind Differencing Scheme for the Time-Accurate Incompressible Navier-Stokes Equations," AIAA Paper, AIAA-1988 88-2583, 1988.

${ }^{8}$ Travin A., Shur M., Strelets M., Spalart P., "Physical and Numerical Upgrades in the Detached-Eddy Simulation of Complex Turbulent Flows," Euromech Colloquium 412 "LES of Complex Transitional and Turbulent Flows", Munich, Germany, October 5-6, 2000. R. Friedrich and W. Rodi (eds.), Kluwer, 2002.

${ }^{9}$ Jenkins, L. N., Neuhart, D. H., McGinley, C. B., Choudhari, M. M., and Khorrami, M. R., "Measurements of Unsteady Wake Interference Between Tandem Cylinders,” AIAA Paper, AIAA-2006-3202.

${ }^{10}$ Spalart, P. R., "Young-person's guide to Detached-Eddy Simulation Grids," NASA-CR 211032, 2001. http://ntrs.nasa.gov/archive/nasa/casi.ntrs.nasa.gov/20010080473 2001126451.pdf [Cited May 30, 2010].

${ }^{11}$ Spalart, P. R., Strelets, M. Kh., Garbaruk, A. V., "Grid Design and the Fate of Eddies in External Flows," In: Proc. of Workshop on Quality and Reliability of Large-Eddy Simulations II, 9-11 September, 2009, Pisa, Italy.

${ }^{12}$ Travin, A., Shur, M., Strelets, M, Spalart, P.R., "Detached-Eddy Simulations past a circular cylinder," J. Flow, Turbulence and Combustion, Vol. 63, 1999, pp. 293-313. 


\section{Argonne}

Leadership Computing Facility Division

Argonne National Laboratory

9700 South Cass Avenue, BIdg. 240

Argonne, IL 60439-4847

www.anl.gov 